Recent Insights into the Physics of the Sun and Heliosphere:

Highlights from SOHO and Other Space Missions

IAU Symposium, Vol. 203, 2001

P. Brekke, B. Fleck, and J. B. Gurman eds.

\title{
Solar Flare Observations at Submm-waves
}

P. Kaufmann ${ }^{1,2}$, J.-P.Raulin ${ }^{1}$, E.Correia ${ }^{1,3}$, J. E.R. Costa ${ }^{1,3}$, C. G. Giménez de Castro ${ }^{1}$, A. V.R. Silva ${ }^{1}$

${ }^{1}$ CRAAM/CRAAE, Universidade Presbiteriana Mackenzie, São Paulo;

${ }^{2}$ Unicamp, Campinas; ${ }^{3}$ Inpe, São José dos Campos, Brazil

H. Levato

Complejo Astronómico El Leoncito, San Juan, Argentina

M. Rovira, C. Mandrini, R. Fernández-Borda

Instituto de Astronomia y Física del Espacio, Buenos Aires, Argentina

O. Bauer

Max-Planck-Institut fur extraterrestrische Physik, Garching, Germany

\begin{abstract}
First $405 \mathrm{GHz}$ and $212 \mathrm{GHz}$ solar flare observations were obtained during short campaigns while the new solar submillimeter-wave telescope (SST) was still undergoing adjustments at the CASLEO El Leoncito observatory in the Argentina Andes. We show here preliminary results for a large X1.1 class X-ray event occurred on 2000 March 22, which exhibited a small submm-w continuum response to the slow (minutes) bulk flare emission, and numerous subsecond spikes (100-300 ms), the brightest spikes reaching about 180 and 50 s.f.u. at 405 and $212 \mathrm{GHz}$, respectively.
\end{abstract}

Solar flare observations are nearly unknown in the submm-IR range of wavelengths. Few results limited to time resolution of about one minute have suggested brightness variations of $10-100 \mathrm{~K}$ in active regions without clear flare associations (Clark \& Park 1970; Hudson 1975).

The six SST beams (Kaufmann et al. 1994) are superimposed on a Kitt Peak solar magnetogram (NOAA 2000) shown in Figure 1 (left) at about the time of the event on 2000 March 22. The flare emission light-curves in compressed time scales are shown in Figure 1 (right) for different frequencies. The $\mathrm{H}-\alpha$ telescope is described elsewhere (Bagalá et al. 1999). The bottom plot of Figure 1 (right) shows the rate of submm-w brightest $(\geq 20 \mathrm{~K})$ spikes incidence with time. An example of such a bright spike (labeled A in Figure 1 (right)) is shown in Figure 2 (A) in a five seconds time interval, compared to data obtained tracking a quiet solar region, near its center, in Figure 2 (B), labeled B in Figure 1 (right). The spiky incidence increases drastically after about $1730 \mathrm{UT}$ approximately together with the soft X-ray level. There is a pronounced concentration of brightenings in correspondence to the bulk emissions at X-rays and $\mathrm{H}-\alpha$. Clusters of spikes seem to be added at about $1745 \mathrm{UT}$ and $1820 \mathrm{UT}$ coincident to flares occurring in AR 

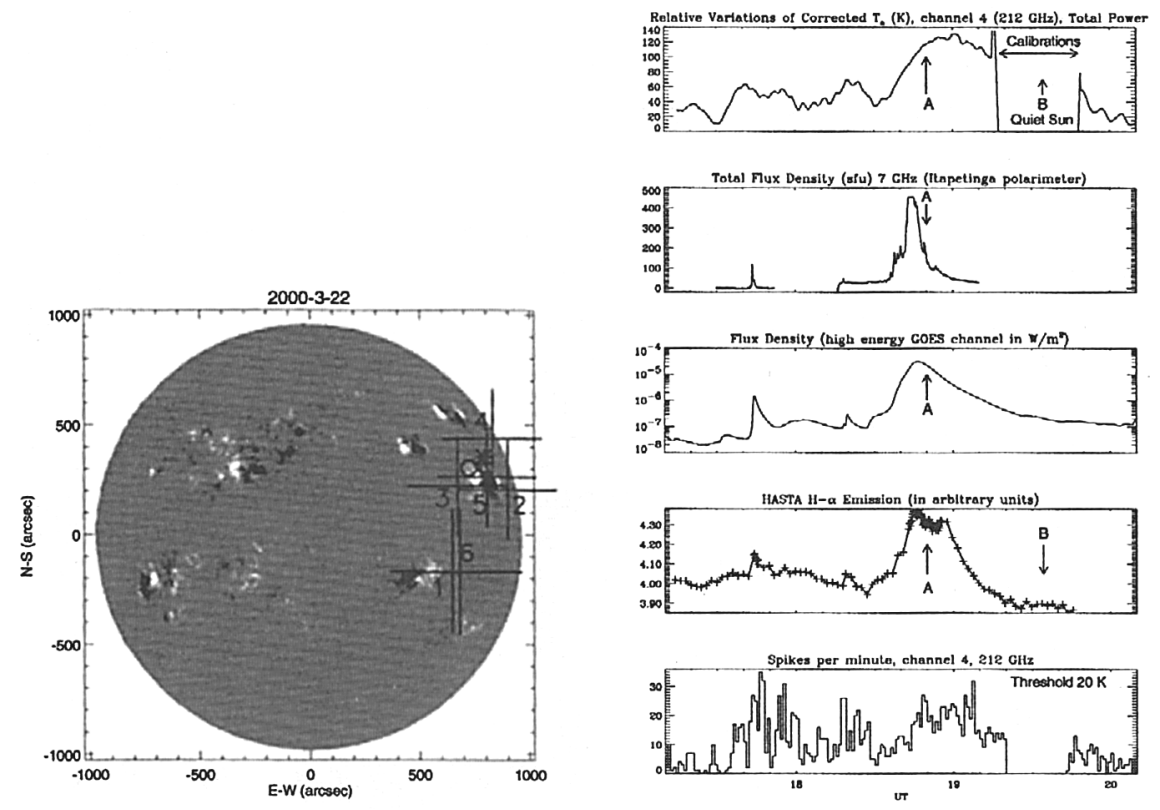

Figure 1. Left: SST beams (1-4 at $212 \mathrm{GHz}, 5-6$ at $405 \mathrm{GHz})$ disposition, with bars indicating the provisional angular half-power sizes. The circle and star indicate the position $\left( \pm 1.5^{\prime}\right)$ for the slow bulk emission at $212 \mathrm{GHz}$ (right, top), and for the bright submm-w spike shown in Fig. $2(\mathrm{~A})$, respectively. Right: Flare time history at different frequencies, from top to bottom: $212 \mathrm{GHz}, 7 \mathrm{GHz}$, GOES soft X-rays, $\mathrm{H}-\alpha$, and $212 \mathrm{GHz}$ spike occurrence rate.

8917 which is still observable by the SST beams. The correlation of observed temperatures in beams 2,3 and 4 allows the approximate determination of the burst location (Giménez de Castro et al. 1999 and references therein), shown by a cross symbol in Figure 1 (left), for which the antenna temperatures are of about $70 \mathrm{~K}$ and $80 \mathrm{~K}$ at $212 \mathrm{GHz}$ and $405 \mathrm{GHz}$, respectively. From the provisional antenna gain we obtain for the spike shown in Figure 2 (A) fluxes of about 50 and 180 s.f.u $( \pm 20 \%)$ at 212 and $405 \mathrm{GHz}$, which corresponds to a positive spectral index of about 2 .

The rapid (100-300 ms) submm-w bright spikes with positive spectral index $(\sim 2)$ bring difficult constraints for thermal mechanisms interpretation (Beckman 1968; Ohki \& Hudson 1975; Kaufmann et al. 1986; McClements \& Brown 1986). They might be an indication of optically thick submm-w spectrum of synchrotron emission from highly relativistic electrons ( $\geq 10 \mathrm{MeV}$ for a field of 1000 gauss), with a spectral turnover frequency possibly in the far infrared (Stein \& Ney 1963; Shklovsky 1964; Kaufmann et al. 1986).

The production of numerous spikes on large active regions and flares has been known at mm- and cm-waves (Kaufmann et al. 1980; Zodi et al 1984; Raulin et al. 1998; Nakajima 2000). The submm-w brightenings bring new essential information for the understanding of elementary energy production in 

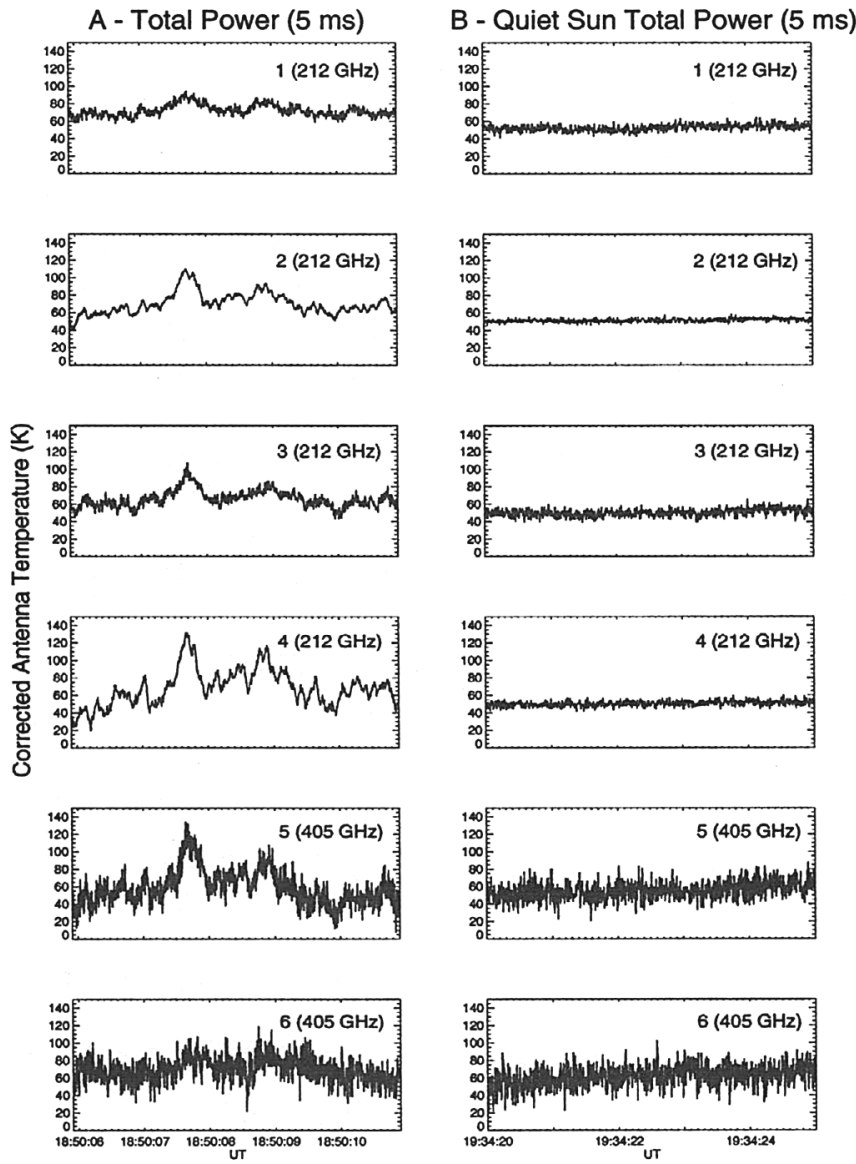

Figure 2. (A) example of a strong submm-w brightening observed by the six SST channels, in units of corrected antenna temperature, with a $5 \mathrm{~ms}$ time constant, compared to (B) an example of a quiet region tracking, near the Sun center.

solar flaring plasmas, in connection to microflares, waves or quakes produced at various time rates, which might have an important role in the heating of the corona (Sturrock \& Uchida 1981; Lin et al. 1984, Zharkova \& Kosovichev 2000, Wang et al. 2000; Phillips 2000). A more complete study of this event is being published elsewhere (Kaufmann et al. 2000).

\section{References}

Bagalá, L.G., Bauer, O.H., Fernández-Borda, R., Francile, C., Haerendel, G., Rieger, E. \& Rovira, M. 1999, ESA SP-448,469.

Beckman, J.E. 1968, Nature 220, 52.

Clark, C.D. \& Park, W.M. 1968, Nature 219, 922. 
Giménez de Castro, C.G., Raulin, J.-P., Makhmutov, V.S., Kaufmann, P. \& Costa, J.E.R. 1999, A\&AS, 140, 373.

Hudson, H.S. 1975, Solar Phys. 45, 69.

Kaufmann, P., Strauss, F.M., Opher, R. \& Laporte, C. 1980, A\&A, 87, 58.

Kaufmann, P., Correia, E., Costa, J.E.R. \& Zodi Vaz, A.M. 1986, A\&A, 157, 11.

Kaufmann, P., Parada, N.J., Magun, A., Rovira, M., Ghielmetti, H. \& Levato, H. 1994, in Proceed. Kofu Symp., NRO Report 360 (Ed. S. Enome \& T. Hirayama), 323

Kaufmann, P., Raulin, J.-P., Correia, E., Costa, J.E.R., Giménez de Castro, C.G., Silva, A.V.R., Levato, H., Rovira, M., Mandrini, C., FernándezBorda, R. \& Bauer, O.H. 2000, ApJ, (submitted)

Lin, R.P., Schwartz, R.A., Kane, S.R., Pelling, R.M. \& Hurley, K.C. 1984, ApJ, $283,421$.

McClements, K.G. \& Brown, J.C. 1996, A\&A, 165, 235.

Nakajima, H. in "High energy solar physics: anticipating Hessi" (Ed. by R. Ramaty \& N. Mandzhavidze), ASP Conf. Series 206,313.

NOAA, Space Environment Center, Solar Geophysical Data reports for March (2000).

Ohki, K. \& Hudson, H.S 1975, Solar Phys. 43,405.

Phillips, K.J.H. 2000, these proceedings.

Raulin, J.-P., Kaufmann, P., Olivieri, R. Correia, E., Makhmutov, V.S. \& Magun, A. 1999, ApJ, 498, L173.

Shlovsky, J. 1964, Nature 202, 275.

Stein, W.A. \& Ney, E.P. 1963, J. Geophys. Res. 68,65.

Sturrock, P.A. \& Uchida, Y. 1981, ApJ, 246, 331.

Wang, H., Qiu, J., Denker, C., Spirock, T.J., Chen, H. \& Goode, P.R. 2000, ApJ, (in press).

Zharkova, V.V. \& Kosovichev, A.G. 2000, in "High energy solar physics: anticipating Hessi" (Ed. by R. Ramaty \& N. Mandzhavidze), ASP Conf. Series 206,77.

Zodi, A.M, Kaufmann, P. \& Zirin, H. 1984, Solar Phys. 92, 283. 